\title{
Disminución de la resistencia mecánica de madera laminada ocasionada por su exposición al fuego
}

\author{
Decrease in the mechanical strength of laminated wood \\ caused by its exposure to fire
}

Javier R. Sotomayor-Castellanos ${ }^{1, *}$ y Gerardo Gallegos-León ${ }^{1}$

Recibido: 07 marzo 2019 | Aceptado: 12 junio 2019 | Publicado en línea: 30 junio 2019 Citación: Sotomayor-Castellanos, JR; Gallegos-León, G. 2019. Disminución de la resistencia mecánica de madera laminada ocasionada por su exposición al fuego. Revista Forestal del Perú 34(1): 66-82. DOI: http://dx.doi.org/10.21704/rfp.v34i1.1286

\begin{abstract}
Resumen
La madera laminada es la tendencia en ingeniería de la madera para sustituir a la madera sólida. El conocimiento de su reacción al fuego tiene aplicación en el diseño arquitectural y en el cálculo para edificar con madera. El objetivo de la investigación fue determinar la disminución de la resistencia mecánica de la madera laminada ocasionada por su exposición al fuego. Se fabricaron piezas de madera laminada de Pinus pseudostrobus y se desarrolló un protocolo de laboratorio específico para medir la disminución de la masa por el fuego, así como para estimar la variación de los módulos de elasticidad estático y de ruptura determinados en pruebas de flexión estática. Se calcularon la densidad y el contenido de humedad de la madera. Para fines del diseño experimental, el factor de variación fue el tiempo de exposición al fuego. Las variables estudiadas fueron: la pérdida de masa, el módulo de elasticidad y el módulo de ruptura. Los parámetros determinados correlacionan estadísticamente de manera importante con el tiempo durante el periodo medido. Los resultados mostraron que la pérdida de masa aumentó con la exposición al fuego. En contraste, los módulos de elasticidad y de ruptura de la madera disminuyeron a medida que el tiempo aumentó.
\end{abstract}

Palabras clave: ingeniería de la madera, madera laminada, densidad de la madera, módulo de elasticidad, módulo de ruptura, pérdida de masa

\footnotetext{
${ }^{1}$ Universidad Michoacana de San Nicolás de Hidalgo, Avenida Francisco J. Múgica S/N Ciudad Universitaria, C.P. 58030, Morelia, Michoacán, México.

* Autor de Correspondencia: madera999@yahoo.com
} 


\begin{abstract}
Laminated wood is the trend in wood engineering to replace solid wood. The knowledge of his reaction to fire has application in the architectural design and calculation to build with wood. The objective of the research was to determine the decrease in the mechanical resistance of laminated wood caused by its exposure to fire. Pieces of laminated wood of Pinus pseudostrobus were manufactured and a specific laboratory protocol was developed to measure the mass reduction by fire, as well as to estimate the variation of the static and rupture elastic moduli determined in static bending tests. The density and moisture content of the wood were calculated. For experimental design purposes, the variation factor was the time of exposure to fire. The variables studied were: mass loss, modulus of elasticity and modulus of rupture. The determined parameters correlate statistically in an important way with the time during the period measured. The results showed that mass loss increased with exposure to fire. In contrast, the modulus of elasticity and breaking of wood decreased as time increased.
\end{abstract}

Key words: wood engineering, laminated wood, wood density, modulus of elasticity, modulus of rupture, loss of mass

\section{Introducción}

El empleo de madera laminada como sustituto de la madera aserrada es la tendencia contemporánea en ingeniería de la madera. Esta tecnología es útil para fabricar elementos estructurales, como lo son vigas y columnas (Nadir y Nagarajan 2014). El conocimiento de su reacción al fuego tiene aplicación práctica en el diseño arquitectural (Laguarda y Espinoza 2015) y en el cálculo ingenieril para la edificación con madera (Lineham et al. 2016).

El módulo de elasticidad, que representa la magnitud de la pendiente en el dominio lineal del diagrama de carga-deformación, y el módulo de ruptura, que es la medida del esfuerzo de flexión en el momento de la ruptura, son dos parámetros de resistencia mecánica necesarios para el diseño y cálculo de estructuras de madera (Polocoșer et al. 2017). Las investigaciones orientadas a medir la reducción de los módulos de elasticidad y de ruptura en la madera preconizan la observación de la temperatura para observar la disminución del valor de estos parámetros (Čekovská et al. 2017, Östman et al. 2017).

Existe poca información que documente la variación de los módulos de elasticidad y de ruptura de piezas de madera laminada con relación al tiempo de exposición al fuego (Bednarek et al. 2009, Lineham et al. 2016).
Una forma de evaluar la reacción al fuego de una pieza de madera es medir la pérdida de masa que sufre después de ser sometida al fuego (Čekovská et al. 2017). La reducción en su resistencia mecánica resultante está relacionada, entre otros factores, con la reducción de la sección transversal cuando se quema la pieza en cuestión (Friquin 2011).

Los métodos y pruebas para evaluar el comportamiento de la madera al fuego adaptan protocolos experimentales y modelos analíticos que simplifican las condiciones reales de incendio. Esto pone en duda su efectividad para predecir la respuesta de una pieza de madera cuando ocurre un accidente donde el fuego está implicado (Brandon et al. 2016). No obstante, y precisamente derivadas de la experimentación, existen referencias sobre los factores que influyen en el fenómeno del fuego y su capacidad para deteriorar la madera. Esta información está detallada para diferentes productos, tipos y dimensiones de probetas.

Por una parte, existen reportes sobre el comportamiento de la madera en caso de incendios en edificaciones con madera (Gernay y Franssen 2015, Bartlett et al. 2017, Östman et al. 2017). Por otra parte, están los resultados derivados de pruebas normalizadas y realizadas en piezas con dimensiones de empleo (Kinjo et al. 2016, Wiesner y Bisby 2018). Otras investi- 
Disminución de la resistencia mecánica de madera laminada ocasionada por su exposición al fuego

gaciones están orientadas al estudio de vigas de madera laminada (Ni y Qiu, 2012, Lineham et al. 2016), mientras que otros autores reportan resultados para tableros empleados en la edificación con madera (Mačiulaitis et al. 2013, Chan-Hom et al. 2017, Cueff et al. 2017). Una perspectiva diferente y complementaria para el estudio de la madera y el fuego es la formulación de modelos reales y a escala de edificaciones en madera ( $\mathrm{Li}$ et al. 2014, Leško y Lopušniak 2016, Östman et al. 2017).

Por su parte, Enberley et al. (2017) recomiendan las pruebas especialmente diseñadas para maderas utilizadas en diseños específicos, así como para nuevas especies o productos compuestos de madera con expectativas de uso comercial. Más cercanos al objeto de estudio de esta investigación están los resultados de pruebas en probetas de pequeñas dimensiones (Mačiulaitis et al. 2012, Almeida et al. 2014, Čekovská et al. 2017) así como la información de modelos analíticos y numéricos (Östman et al. 2017, Zhao et al. 2017) sobre la caracterización del fenómeno.

A propósito de esta última perspectiva, las conclusiones de los trabajos anteriormente citados, y pertinentes para la presente investigación, se pueden resumir en las siguientes proposiciones:

1. Existen métodos estandarizados para el estudio del comportamiento de la madera en relación con su exposición al fuego. Además, los investigadores desarrollan ensayos ad-hoc asociados con modelos analíticos y/o numéricos para estudiar aspectos particulares del fenómeno; de tal forma que los resultados entre experimentos son difíciles de comparar. Así, para emitir un juicio, se recomienda considerar caso por caso y tomar en cuenta las condiciones experimentales especificadas en cada investigación.

2. Las variables estudiadas con mayor frecuencia son: tiempo de ignición, pérdida de masa, determinación de la capa cero y de la velocidad de carbonizado. La mayoría de los experimentos miden estos parámetros en función de la temperatura de exposición.
3. El modo en que la madera reacciona al fuego se diferencia de su resistencia, cuando es expuesta al fuego. A este respecto, es poca la información sobre mediciones directas de la disminución de los módulos de elasticidad y de ruptura de probetas de madera, en función del tiempo de exposición al fuego.

Por otra parte, la literatura reporta que los módulos de elasticidad y de ruptura de la madera laminada disminuyen cuando es expuesta al fuego (Schmid et al. 2014). Este fenómeno está relacionado con la perspectiva de la reducción de propiedades. Desde esta perspectiva, se evalúa el comportamiento al fuego de una pieza de madera empleando el método de la sección transversal efectiva, asociado a su vez con el enfoque de la capa de resistencia cero (König, 2009, Hopkin et al. 2011, Schmid et al. 2014; Schmid et al. 2015, Brandon et al. 2016). De esta manera, se actualiza el paradigma vigente en ingeniería de la madera el cual sostiene que, después de un periodo de exposición al fuego, la madera crea una capa de madera carbonizada que actúa como protección y aislante, de tal forma que desacelera el proceso de pirolisis de la madera (Xu et al. 2015, Rocha y Landesmann 2016, Emberley et al. 2017).

La madera de $P$. pseudostrobus es utilizada ampliamente en la industria de productos forestales (Sáenz et al. 2011). Sus características mecánicas están reportadas por Sotomayor (2015a) y Sotomayor (2015b). Para la madera laminada de esta especie, Sotomayor (2016) y Sotomayor (2017) reportan sus módulos dinámicos determinados por métodos no destructivos.

A manera de hipótesis de trabajo, en esta investigación se propone que las magnitudes de los módulos de elasticidad y de ruptura disminuyen cuando una pieza de madera es expuesta al fuego. Para verificar esta hipótesis, el objetivo de la investigación fue medir la disminución de la resistencia mecánica de madera laminada ocasionada por su exposición al fuego. Para lograr este objetivo, se fabricaron piezas de madera laminada de Pinus pseudos- 
trobus Lindl. Asimismo, se desarrolló un protocolo de laboratorio específico para medir la disminución de la masa por el fuego, así como estimar la variación de los módulos de elasticidad estático y de ruptura, determinados en pruebas de flexión estática. Este fenómeno se observó durante un periodo de tiempo de exposición de doce minutos.

\section{Materiales y métodos}

\section{Diseño experimental}

La unidad experimental consistió en 35 probetas de madera laminada de $P$. pseudostrobus, agrupadas en siete muestras independientes, compuestas por cinco probetas cada una. Este material es el mismo empleado por Sotomayor et al. (2015). La exposición al fuego de la madera se consideró el tratamiento. El factor de variación fue el tiempo de exposición al fuego $\left(\mathrm{t}_{\exp }\right)$ con siete niveles: $0,2,4,6,8,10$ y 12 minutos. Las variables de respuesta fueron la pérdida de masa $(\Delta \mathrm{m})$, el módulo de elasticidad (MOE) y el módulo de ruptura (MOR). La densidad aparente de la madera laminada $\left(527 \mathrm{~kg} . \mathrm{m}^{-3}\right)$, así como su contenido de humedad (14,5\%) se consideraron parámetros de referencia invariables que no intervinieron en el experimento.

Para cada variable de cada una de las siete muestras se calcularon media $(\overline{\mathbf{x}})$, desviación estándar $(\sigma)$ y coeficiente de variación en por ciento $(C V=\sigma / \bar{x})$. Se determinó la normalidad en la distribución de las muestras con el criterio de demarcación para el sesgo estandarizado (SE) y el apuntamiento estandarizado (AE): [-2 $<\mathrm{SE}$ y/o AE <+2]; asimismo, se verificó la igualdad de varianzas con la hipótesis: $\mathrm{H}_{0}: \sigma_{1}=\sigma_{2}$ con un nivel de significancia de $5 \%(\alpha=0,05)$. A continuación, se realizó un análisis de varianza con la hipótesis nula: $\mathrm{H}_{0}: \overline{\mathbf{x}}_{1}=\overline{\mathbf{x}}_{2}$ con un nivel de significancia de $5 \%(\alpha=0,05)$. El criterio de demarcación fue aceptar una diferencia estadísticamente significativa para valores $P>$ 0,05 . Cuando procedió rechazar la hipótesis nula, se efectuaron pruebas de rango múltiples.

Para analizar las tendencias de las variables de respuesta se calcularon las regresiones lineales $(\mathrm{y}=\mathrm{ax}+\mathrm{b})$ y sus coeficientes de determinación $\left(\mathrm{R}^{2}\right)$, entre $\Delta \mathrm{m}, \mathrm{MOE}$ y MOR como variables dependientes del tiempo de exposición $\left(\mathrm{t}_{\text {exp }}\right)$, como variable independiente. Complementando, se calcularon las regresiones lineales entre MOE y MOR en función de $\Delta \mathrm{m}$.

\section{Materiales}

Se recolectaron piezas de madera aserrada de Pinus pseudostrobus Lindl. var. pseudostrobus, en un aserradero de la región de Ciudad Hidalgo ( $\left.19^{\circ} 41^{\prime} 30^{\prime \prime} \mathrm{N}-100^{\circ} 33^{\prime} 13^{\prime \prime} \mathrm{O}\right)$, Michoacán. Las piezas de madera tenían dimensiones comerciales y se adquirieron en estado seco por estufado. Las probetas de madera de $P$. pseudostrobus se elaboraron de acuerdo al procedimiento reportado por Sotomayor et al. (2015). A partir de las piezas de madera aserrada, se prepararon 175 tabletas con sección transversal de 0,05 $\mathrm{m}$ de ancho, 0,01 $\mathrm{m}$ de espesor y de 0,8 $\mathrm{m}$ de largo. Estas dimensiones correspondieron a las direcciones radial, tangencial y longitudinal del plano leñoso.

La madera se conservó en una cámara de acondicionamiento con una temperatura de 20 ${ }^{\circ} \mathrm{C}\left( \pm 1^{\circ} \mathrm{C}\right)$ y una humedad relativa del aire de $65 \%( \pm 2 \%)$, hasta que su peso fue constante.

Para adherir las tabletas, se aplicaron 2,5 $\mathrm{kg} \cdot \mathrm{m}^{-2}$ de pegamento de contacto a base de resina de poliacetato de vinilo, repartidos en las cuatro caras interiores de las viguetas, correspondientes al plano longitudinal-radial (Figura 1). Una vez armadas las probetas, se posicionaron en un dispositivo ad-hoc y se prensaron en la dirección tangencial, hasta que las viguetas alcanzaron una altura o espesor uniforme de $0,053 \mathrm{~m}$. El ancho de las viguetas se ajustó a 0,049 $\mathrm{m}$ y el largo a 0,79 $\mathrm{m}$. Con el objetivo de que el adhesivo solidificara, el tiempo de prensado fue de 48 horas en ambiente de laboratorio (Temperatura de $20{ }^{\circ} \mathrm{C}$ y humedad relativa del aire de 65\%). Finalmente, las probetas de madera laminada, se almacenaron durante un mes en la cámara de acondicionamiento con las condiciones de temperatura y de humedad relativa del aire antes citadas, hasta que su peso fue constante. 


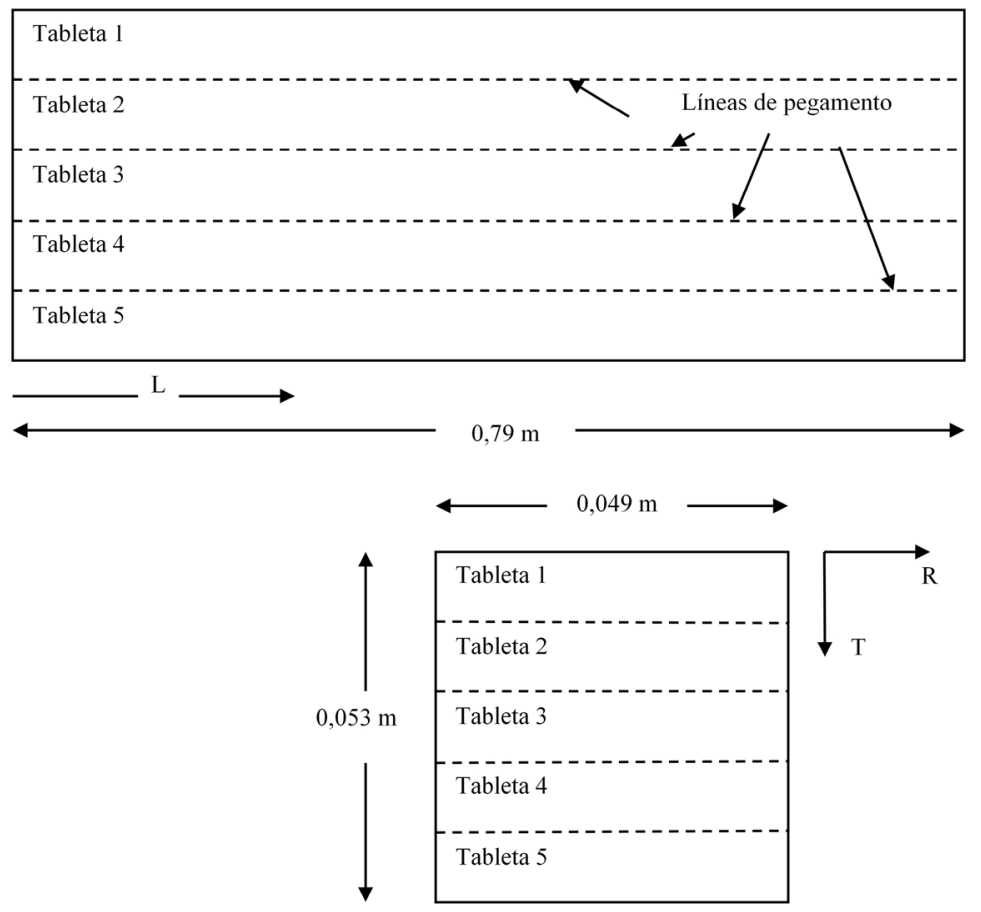

Figura 1. Diagrama de la estructura de las probetas (tomado de Sotomayor et al. 2015).

\section{Métodos}

El contenido de humedad de la madera $(\mathrm{CH})$ se calculó con la relación del peso húmedo de la probeta al momento del ensayo y su peso en estado anhidro, adaptando la norma ISO 13061-1:2014.

La densidad aparente de la madera al momento del ensayo $\left(\rho_{\mathrm{CH}}\right)$ correspondiente a un contenido de humedad $(\mathrm{CH})$, se calculó con la relación peso - volumen, adaptando la norma ISO 13061-2:2014.

\section{Pruebas de reacción al fuego}

Las pruebas de reacción al fuego se realizaron adaptando los protocolos reportados por Elvira-León et al. (2016), Čekovská et al. (2017) y Sotomayor y Gallegos (2017). Estos protocolos consisten en posicionar la probeta en el dispositivo para las pruebas de reacción al fuego, colocado al interior de una campana de laboratorio (Figura 2), y exponerla a la flama de 0,09 $\mathrm{m}$ de altura, producida por un mechero Meker-Fisher con regulación de aire y de gas licuado de petróleo, con tapa de rejilla de $0,03 \mathrm{~m}$ de diámetro y con una temperatura máxima de $1300^{\circ} \mathrm{C}$. La probeta se posiciona en dos soportes, de tal forma que la flama incide perpendicularmente en la dirección longitudinal de la probeta. La madera se expone a seis tiempos de exposición: dos, cuatro, seis, ocho, diez y doce minutos. El tiempo de exposición cero se refiere a que la probeta no es expuesta al fuego.

\section{Pérdida de masa}

La pérdida de masa de la probeta ocasionada por la exposición al fuego se calculó con la fórmula (1) (Yuksel et al. 2014):

$$
\Delta \mathrm{m}=\left(\frac{\mathrm{P}_{\mathrm{a}}-\mathrm{P}_{\mathrm{d}}}{\mathrm{Pa}_{\mathrm{a}}}\right) \times 100
$$




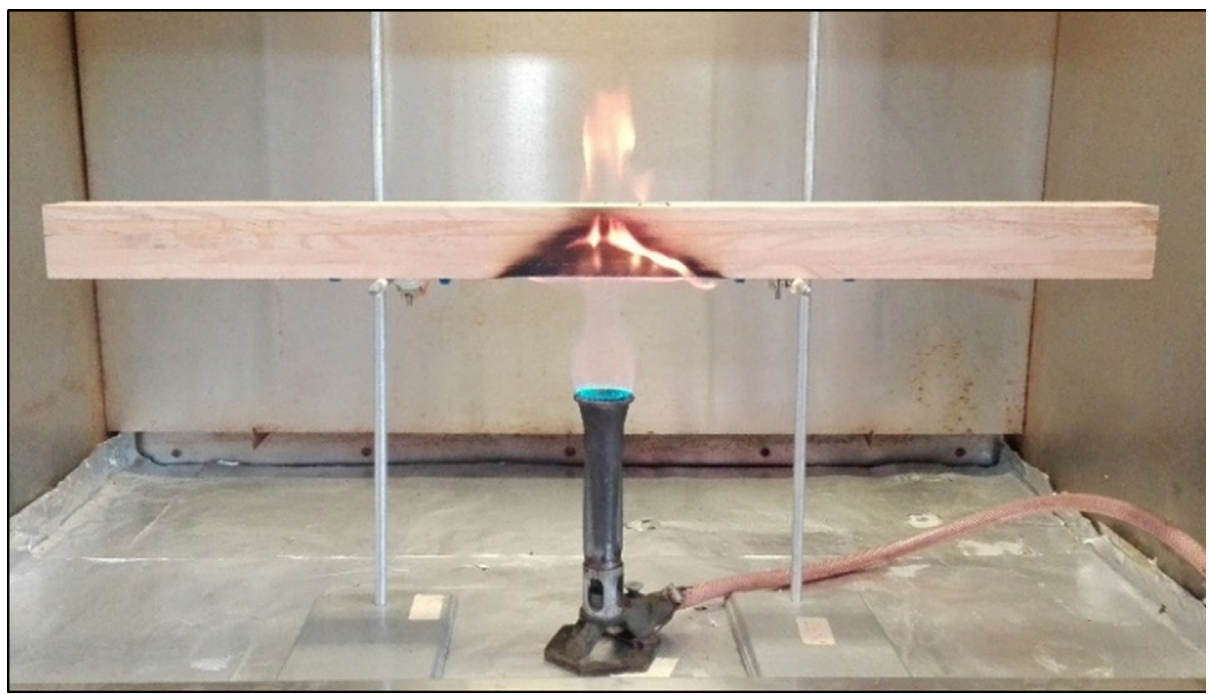

Figura 2. Pruebas de reacción al fuego.

Donde:

$\Delta \mathrm{m}=$ Pérdida de masa $(\%)$

$\mathrm{P}=$ Peso de la probeta antes de la exposición al fuego $(\mathrm{kg})$

$\mathrm{P}_{\mathrm{d}}=$ Peso de la probeta después de la exposición al fuego $(\mathrm{kg})$

\section{Pruebas de flexión estática}

Las pruebas de flexión estática consistieron en aplicar una carga transversal (P) a la dirección longitudinal de la probeta en medio de su portada (L) y sobre la cara opuesta a la de la zona carbonizada (Figura 3). La velocidad de carga fue de 150 N.min ${ }^{-1}$. Se utilizó una máquina universal de ensayos mecánicos Forney Testing Machine ${ }^{\varpi}$ (USA), modelo LT-1150, con capacidad de 150 toneladas. Durante los ensayos, para el dominio elástico se midieron tanto los intervalos de la carga $(\Delta \mathrm{P})$ como los de la deformación $(\Delta y)$ y se elaboraron diagramas carga-deformación (Figura 4). Cuando la probeta falló, se midió la carga a la ruptura $\left(\mathrm{P}_{\text {rup }}\right)$.

El módulo de elasticidad se calculó con la fórmula (2) (Missanjo y Matsumura 2016):

$$
\mathrm{MOE}=\frac{\Delta \mathrm{P}}{\Delta \mathrm{y}} \frac{\mathrm{L}^{3}}{48 \mathrm{I}}
$$

Donde:

MOE = Módulo de elasticidad (N.m²)

$\Delta \mathrm{P}=$ Intervalo de la carga $(\mathrm{N})$

$\Delta \mathrm{y}=$ Intervalo de la deformación (m)

$\mathrm{L}=$ Distancia entre apoyos $(\mathrm{m})$

$\mathrm{I}=$ Momento de inercia de la sección trasver$\mathrm{sal}\left(\mathrm{m}^{4}\right)$

El módulo de ruptura se calculó con la fórmula (3) (Missanjo y Matsumura 2016):

$$
\text { MOR }=\frac{3}{2} \frac{\text { Prup L }}{\mathrm{b} \mathrm{h}^{2}}
$$

Donde:

MOR = Módulo de ruptura $\left(\mathrm{N} . \mathrm{m}^{-2}\right)$

Prup $=$ Carga a la ruptura $(\mathrm{N})$

$\mathrm{L}=$ Distancia entre apoyos $(\mathrm{m})$

$\mathrm{b}=$ Base de la probeta $(\mathrm{m})$

$\mathrm{h}=$ Altura de la probeta $(\mathrm{m})$ 


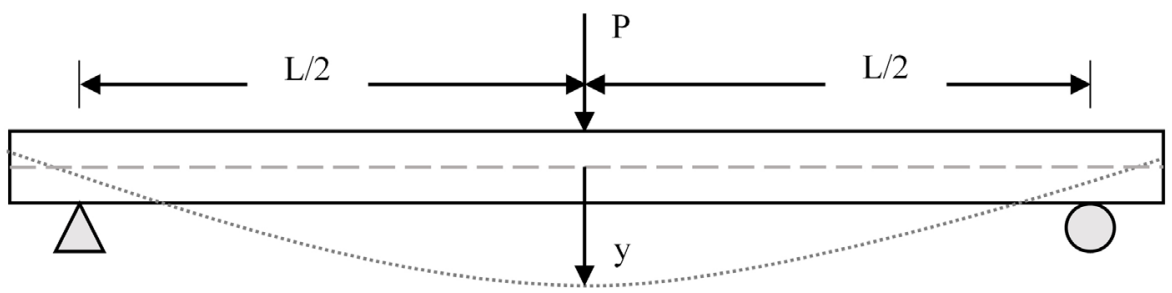

Figura 3. Configuración de las pruebas de flexión.

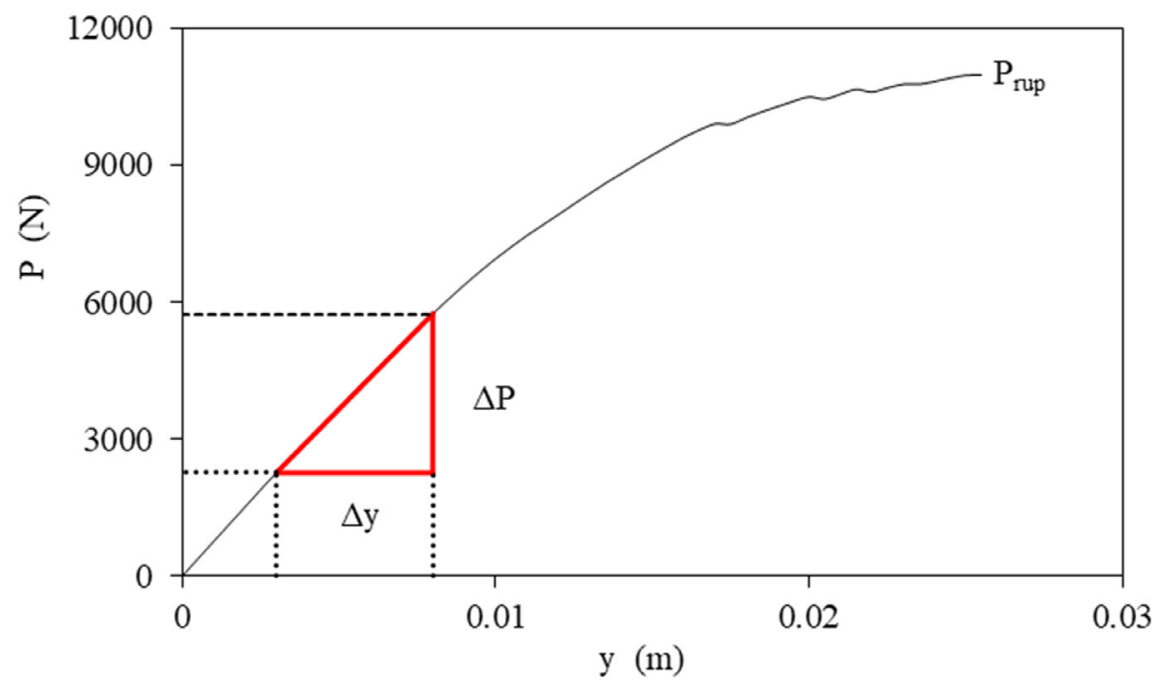

Figura 4. Diagrama carga-deformación.

\section{Resultados}

El Cuadro 1 presenta la pérdida de masa, el módulo de elasticidad y el módulo de ruptura referidos, según el tiempo de exposición. El Cuadro 2 presenta los resultados de las pruebas de normalidad, de verificación y análisis de varianza, así como de homogeneidad.

Para las variables pérdida de masa, módulo de elasticidad y módulo de ruptura, las distribuciones de los resultados de las siete muestras, correspondientes a los tiempos de exposición de $0,2,4,6,8,10$ y 12 minutos, resultaron normales.

Asimismo, la verificación de varianza entre muestras, correspondientes a los seis tiempos de exposición, reporta que no existe entre ellas una diferencia estadísticamente significativa $(P \geq 0,05)$, con un nivel del 95\% de confianza, resultado que valida este análisis. El análisis de varianza $\left(P_{(\alpha=0,05)}<0,05\right)$ reporta que existe entre las muestras una diferencia estadísticamente significativa con un nivel del $95 \%$ de confianza. Es decir, $\Delta \mathrm{m}$ aumenta con la exposición al fuego. En contraste, MOE y MOR disminuyen a medida que el tiempo de exposición aumenta.

Sin embargo, el análisis de grupos homogéneos reporta que para la pérdida de masa correspondiente a los tiempos de exposición 6, 8 y 10 , no existen diferencias estadísticamente significativas entre estas muestras. En cam- 
bio, para el módulo de elasticidad, únicamente los tiempos de exposición de 2 y 4 minutos se diferencian. Para el módulo de ruptura, con excepción de la muestra correspondiente al tiempo de exposición cero, el resto de las muestras no presentan diferencias estadísticamente significativas.

Se observa que los resultados para los tiempos de exposición 0, 2 y 4 se agrupan, al igual que los correspondientes a los tiempos de exposición 6,8 y 10 minutos. El tiempo de exposición 4 se presenta como punto de inflexión. Finalmente, se observa que el tiempo de exposición 12 se diferencia. Esta uniformidad en los resultados sugiere la conveniencia de las pruebas de laboratorio implementadas para provocar la variación de la pérdida de masa, del módulo de elasticidad y del módulo de ruptura.

\begin{tabular}{|c|c|c|c|c|}
\hline$t_{\text {exp }}(\min )$ & Estadístico & $\begin{array}{l}\Delta \mathrm{m} \\
(\%)\end{array}$ & $\begin{array}{c}\text { MOE } \\
\left(\text { MN.m-m }{ }^{-2}\right)\end{array}$ & $\begin{array}{c}\text { MOR } \\
\left(\text { MN.m }{ }^{-2}\right)\end{array}$ \\
\hline \multirow{3}{*}{0} & $\bar{x}$ & 0,09 & 9427 & 88,0 \\
\hline & $\sigma$ & - & 1601 & 2,1 \\
\hline & $\mathrm{CV}$ & - & 17,0 & 2,3 \\
\hline \multirow{3}{*}{2} & $\bar{x}$ & 1,14 & 8455 & 61,8 \\
\hline & $\sigma$ & - & 1461 & 14,6 \\
\hline & $\mathrm{CV}$ & - & 17,3 & 23,6 \\
\hline \multirow{3}{*}{4} & $\bar{x}$ & 1,99 & 6533 & 52,4 \\
\hline & $\sigma$ & - & 853 & 3,1 \\
\hline & $\mathrm{CV}$ & - & 13,1 & 5,9 \\
\hline \multirow{3}{*}{6} & $\bar{x}$ & 2,96 & 6209 & 43,8 \\
\hline & $\sigma$ & - & 462 & 11,6 \\
\hline & $\mathrm{CV}$ & - & 7,4 & 26,5 \\
\hline \multirow{3}{*}{8} & $\bar{x}$ & 3,16 & 6005 & 48,0 \\
\hline & $\sigma$ & - & 801 & 9,3 \\
\hline & $\mathrm{CV}$ & - & 13,3 & 19,3 \\
\hline \multirow{3}{*}{10} & $\bar{x}$ & 3,18 & 5811 & 50,2 \\
\hline & $\sigma$ & - & 810 & 8,7 \\
\hline & $\mathrm{CV}$ & - & 13,9 & 17,3 \\
\hline \multirow{3}{*}{12} & $\bar{x}$ & 4,46 & 5027 & 36,7 \\
\hline & $\sigma$ & - & 811 & 21,0 \\
\hline & $\mathrm{CV}$ & - & 16,1 & 57,3 \\
\hline
\end{tabular}

$\mathrm{t}_{\exp }=$ Tiempo de exposición; $\Delta \mathrm{m}=$ Pérdida de masa; MOE = Módulo de elasticidad; MOR = Módulo de ruptura; = Media; $\sigma=$ Desviación estándar; $\mathrm{CV}=$ Coeficiente de variación porcentual.

Cuadro 1. Tiempo de exposición, pérdida de masa, módulo de elasticidad y módulo de ruptura. 
Disminución de la resistencia mecánica de madera laminada

\begin{tabular}{ccccccc}
\hline & \multicolumn{3}{c}{ Pruebas de normalidad } \\
$\mathrm{t}_{\text {exp }}$ & \multicolumn{2}{c}{$\Delta \mathrm{m}$} & \multicolumn{2}{c}{ MOE } & \multicolumn{2}{c}{ MOR } \\
& $\mathrm{SE}$ & $\mathrm{AE}$ & $\mathrm{SE}$ & $\mathrm{AE}$ & $\mathrm{SE}$ & $\mathrm{AE}$ \\
0 & 1,021 & 0,664 & $-0,865$ & 0,310 & $-0,019$ & $-1,243$ \\
2 & $-1,015$ & 0,183 & $-1,470$ & 1,337 & $-1,500$ & 1,448 \\
4 & 0,392 & $-0,357$ & 1,224 & 0,528 & 0,050 & $-1,050$ \\
6 & 1,328 & 1,150 & 1,632 & 1,599 & $-0,536$ & $-1,234$ \\
8 & $-0,793$ & 0,702 & 1,187 & 0,316 & $-1,421$ & 1,249 \\
10 & 0,983 & 0,469 & $-1,218$ & 0,405 & $-1,801$ & 1,785 \\
12 & $-1,656$ & 1,517 & 1,415 & 1,478 & 1,419 & 0,996 \\
\hline
\end{tabular}

Verificación de varianza

\begin{tabular}{|c|c|c|c|}
\hline & $\Delta \mathrm{m}$ & MOE & MOR \\
\hline Valor $\mathrm{P}_{(\alpha=0.05)}$ & $0,156^{*}$ & $0,682 *$ & $0,458^{*}$ \\
\hline
\end{tabular}

Análisis de varianza

\begin{tabular}{|c|c|c|c|}
\hline & $\Delta \mathrm{m}$ & MOE & MOR \\
\hline Valor $\mathrm{P}_{(\alpha=0,05)}$ & $<0,001$ & $<0,001$ & $<0,001$ \\
\hline
\end{tabular}

\section{Grupos homogéneos}

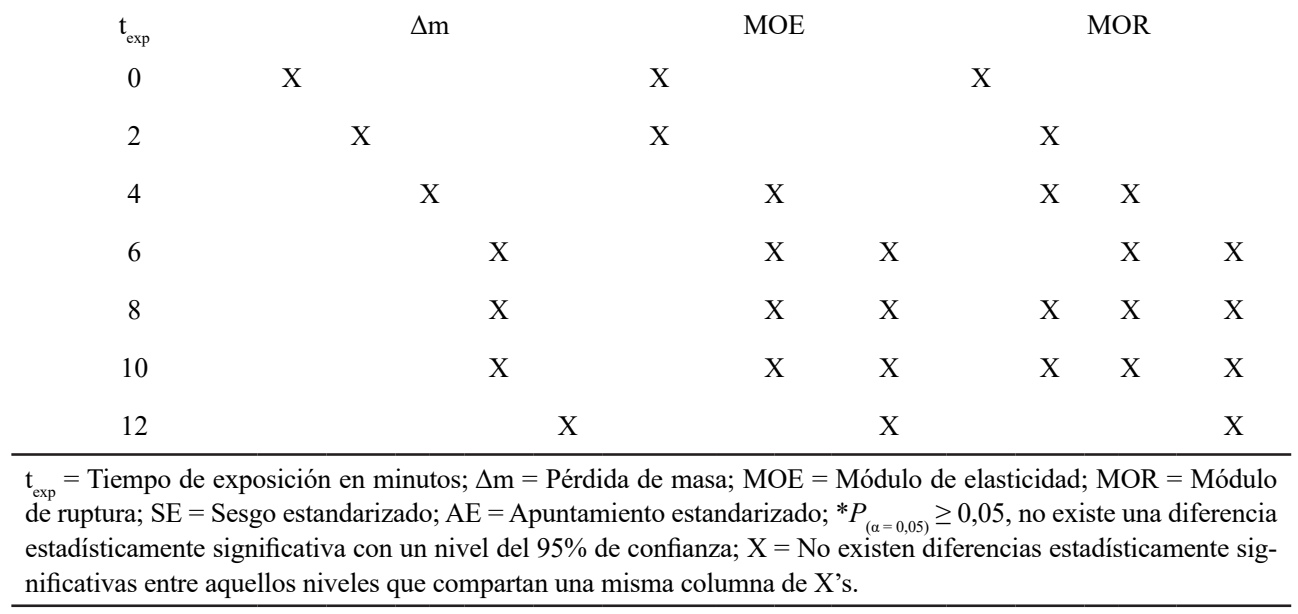

Cuadro 2. Resultados de las pruebas de normalidad, de verificación y análisis de varianza, así como de homogeneidad.

\section{Discusión}

\section{Disminución de la sección transversal}

La sección transversal donde impacta la flama disminuye a medida que se incrementa el tiempo de exposición. La Figura 5 presenta cinco probetas expuestas al fuego durante intervalos que van de cero a doce minutos. Las dimensiones de la sección transversal de las probetas utilizadas en la presente investigación se asemejan a las de piezas de madera aserrada que se emplean en elementos de resistencia en edificaciones con madera. 
Esta disminución se localiza en medio de la portada de las probetas en las pruebas de reacción al fuego (Figura 2) y corresponde al punto medio de la portada de las pruebas de flexión (Figura 3). Es en esta zona donde el momento de flexión y el esfuerzo cortante son máximos.
Esta tendencia de la sección transversal a disminuir, a medida que el tiempo de exposición aumenta, para el caso de la altura (h) es lineal (Figura 6). En cambio, para la base (b), y no obstante que la correlación lineal resulta con un fuerte coeficiente de determinación, se dis-

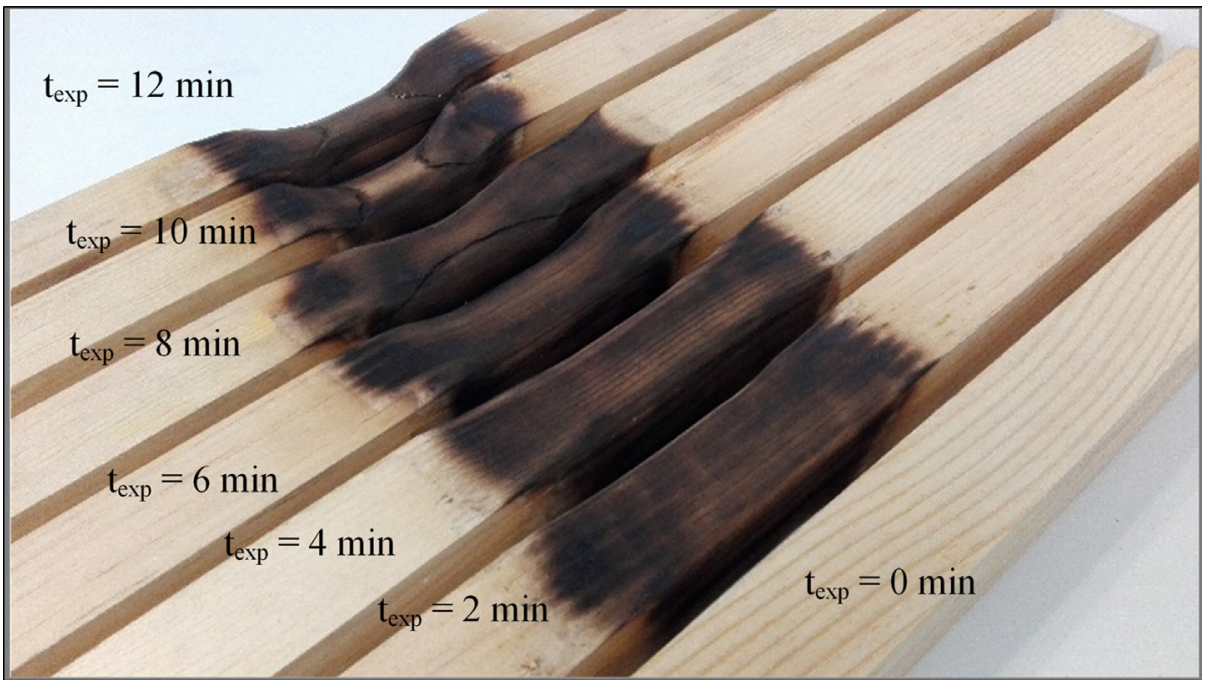

Figura 5. PProbetas después de su exposición al fuego.

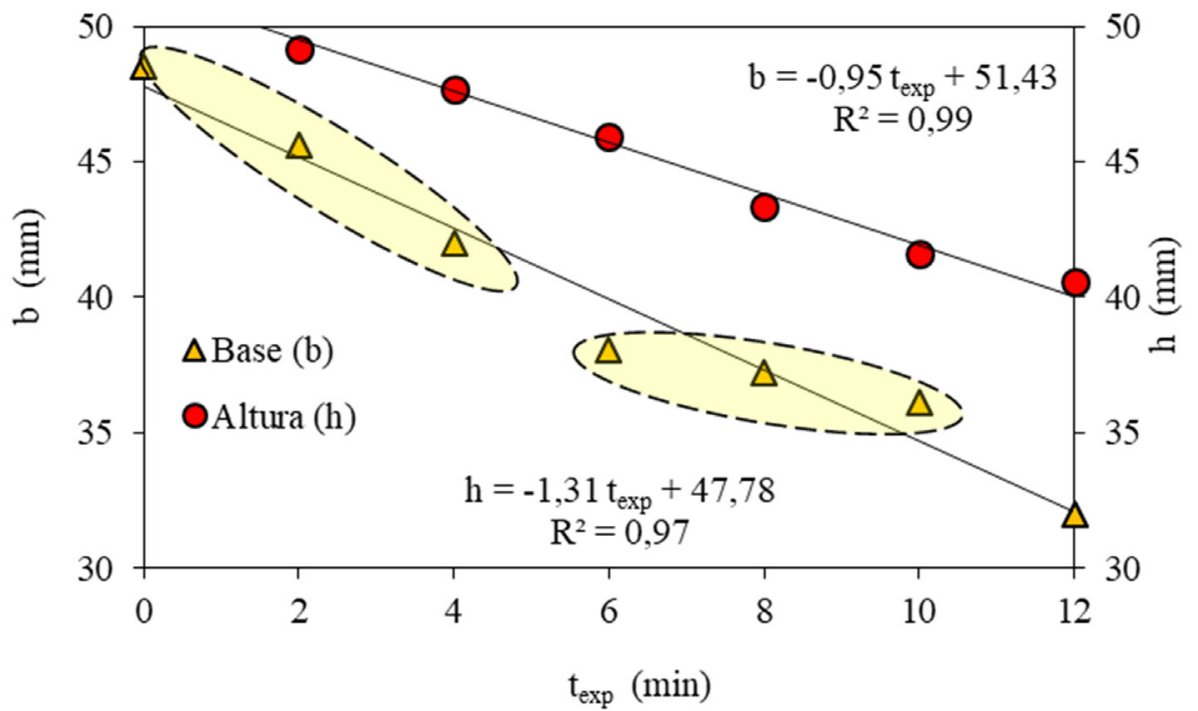

Figura 6. Disminución de la base (b) y de la altura (h) de la sección transversal de las probetas en función del tiempo de exposición $\left(\mathrm{t}_{\exp }\right)$. 
tinguen tres periodos. En el primero, que contiene los tiempos de exposición 0,2 y 4 minutos, la pendiente es mayor comparativamente a la del segundo periodo, que comprende los tiempos de exposición 6,8 y 10 minutos, en el que la disminución desacelera. Finalmente, en el tercer periodo, al minuto 12 , se retoma la tendencia general d disminuir. En esta figura y en las subsecuentes, los puntos experimentales que se muestran en los gráficos significan los valores promedio correspondientes a cada uno de los tiempos de exposición observados.

\section{Pérdida de masa}

La pérdida de masa aumenta proporcionalmente al tiempo de exposición (Cuadro 1). Esta tendencia es lineal y con un fuerte coeficiente de determinación (Figura 7). Sin embargo, $\mathrm{y}$ al igual que en la disminución de las dimensiones de la sección transversal, se observan tres etapas. En la primera, para los tiempos de exposición 0,2 y 4 minutos, se percibe un aumento. Sin embargo, para los tiempos de exposición 6,8 y 10, este crecimiento se desacelera, para finalmente retomar su curso en el tiempo de exposición 12. Este hecho coincide con los resultados del análisis de grupos homogéneos (Cuadro 2), en los que se observa la ausencia de diferencias estadísticamente significativas de pérdida de masa para los tiempos de exposición de 6, 8 y 10 minutos.

Este aumento de la pérdida de masa en la madera laminada de $P$. pseudostrobus, a medida que el tiempo de exposición al fuego aumenta, coincide con los resultados reportados en la bibliografía (Almeida et al. 2014, Xu et al. 2015, Rocha y Landesmann 2016, Emberley et al. 2017). Con todo, estos autores señalan que la cantidad y/o la velocidad de esta disminución de la masa de madera difieren según la especie y/o el tipo de material en estudio, así como de la configuración particular a cada procedimiento experimental realizado. Igualmente, señalan que este parámetro es un indicador de la severidad del efecto del fuego en la madera y su impacto tanto en su estructura anatómica, como en su composición química.

\section{Módulo de elasticidad}

El módulo de elasticidad promedio de las probetas de madera laminada, correspondiente al tiempo de exposición igual a cero, es decir, sin exposición al fuego, fue $28,3 \%$ menor comparativamente con el módulo de elasticidad de madera sólida de $P$. pseudostrobus (MOE $=13141 \mathrm{MN} \cdot \mathrm{m}^{-2} ; \rho_{\mathrm{CH}}=540 \mathrm{~kg} \cdot \mathrm{m}^{-3}$ ) reportada

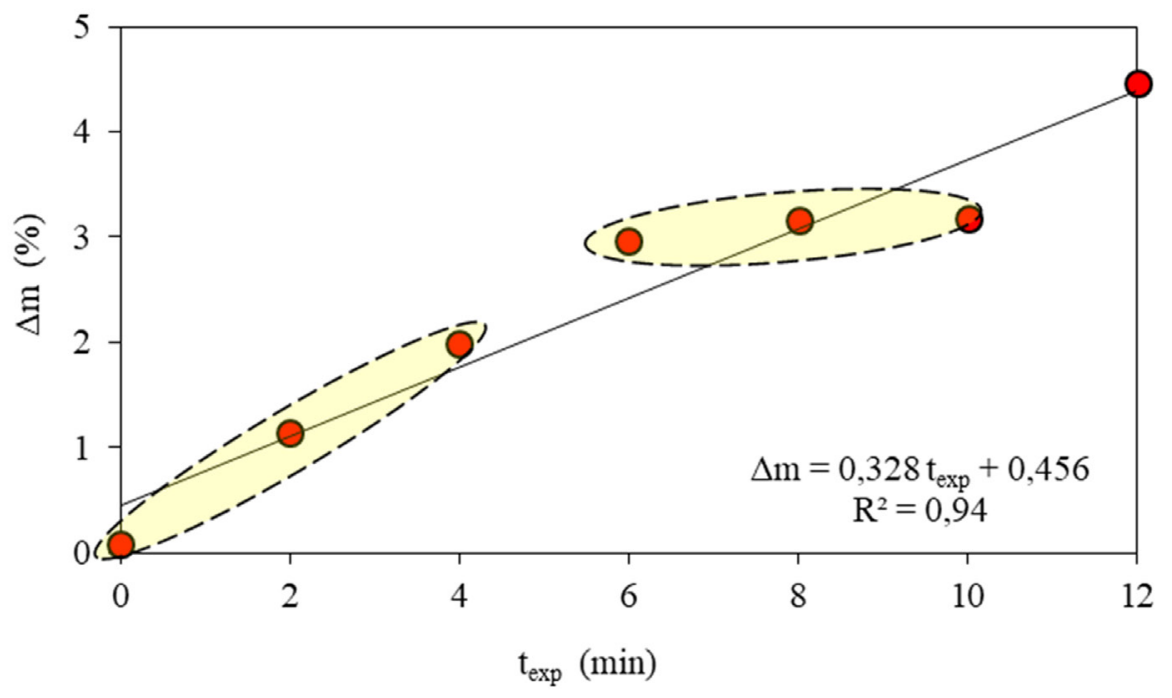

Figura 7. Pérdida de masa $(\Delta \mathrm{m})$ en función del tiempo de exposición $\left(\mathrm{t}_{\exp }\right)$. 
por Sotomayor (2015). En el mismo contexto, el módulo de elasticidad de las probetas de madera laminada se sitúa al interior del intervalo reportado por el Laboratorio de Productos Forestales de los Estados Unidos (Forest Products Laboratory 2010) para madera estructural laminada que va de $9000 \mathrm{MN} \cdot \mathrm{m}^{-2}$ a 14500 MN.m ${ }^{-2}\left(400 \mathrm{~kg} \cdot \mathrm{m}^{-3}<\rho_{\mathrm{CH}}<600 \mathrm{~kg} \cdot \mathrm{m}^{-3}\right)$.
La Figura 8 muestra los diagramas carga-deformación promedio para cada muestra de cinco probetas correspondientes a los tiempos de exposición. En la Figura 8a, se exhiben las curvas completas hasta la carga de ruptura. En la Figura 8b, se detallan las pendientes correspondientes a las zonas elásticas de los diagramas.

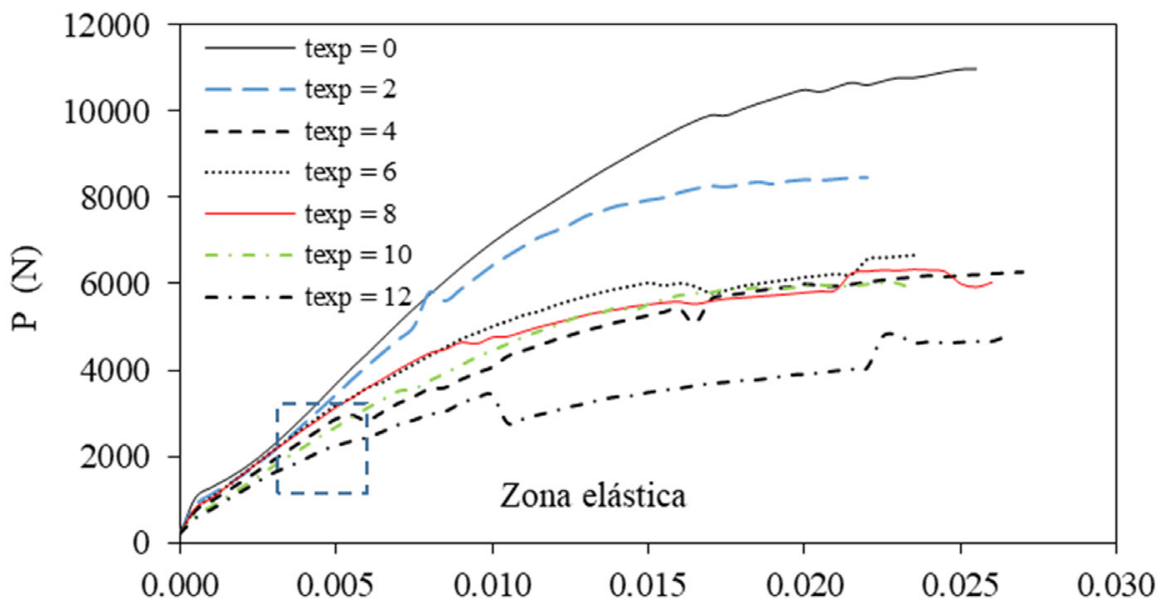

a)

y (m)

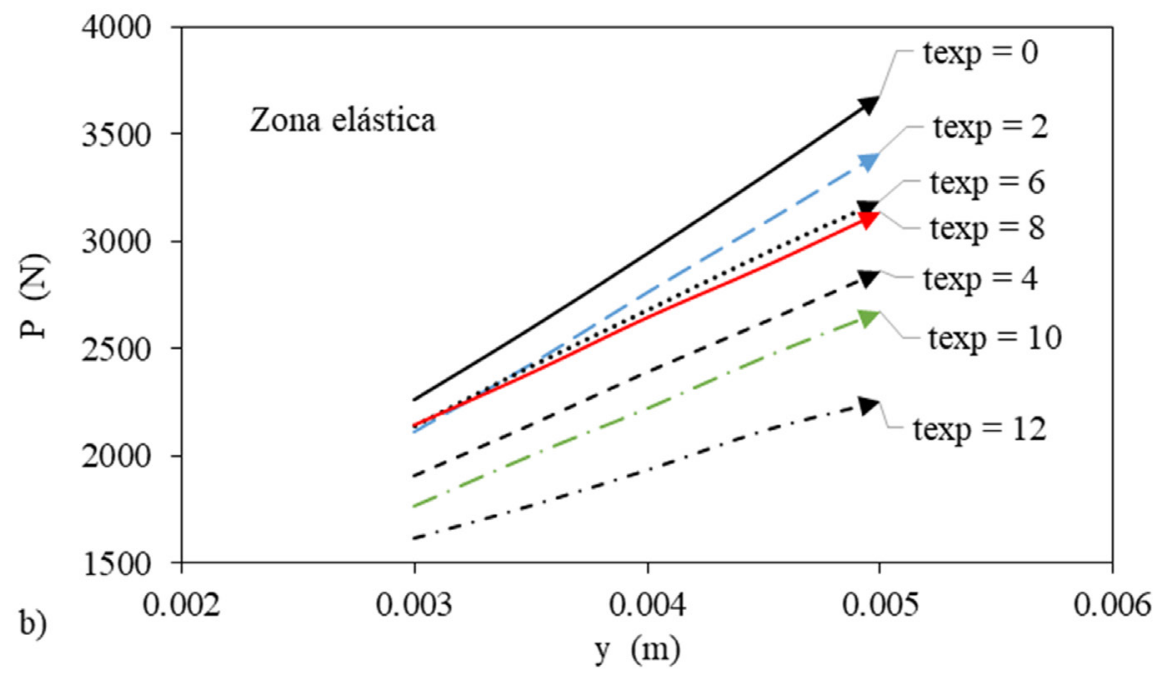

Figura 8. a) Diagramas carga (P)-deformación (y) para diferentes tiempos de exposición (texp) yb) detalles de la zona elástica de los diagramas. 
Disminución de la resistencia mecánica de madera laminada ocasionada por su exposición al fuego

La respuesta de las probetas mostró la tendencia general de disminuir su capacidad de carga a medida que el tiempo de exposición aumenta. Con excepción de las probetas correspondientes al tiempo de exposición cero, durante las pruebas de flexión se observaron fallas antes de que se llegara a la carga de ruptura. Aun así, las probetas siguieron soportando el incremento en la carga, hasta llegar a la ruptura total. Este fenómeno se observa en la discontinuidad en los diagramas carga-deformación presentados en la Figura 8a.

Respecto al tiempo de exposición, las deformaciones máximas al momento de la ruptura de las siete muestras son similares. En cambio, para la capacidad de carga, se distinguen tres grupos. Al grupo de alta resistencia corresponden los tiempos de exposición 0 y 2 minutos, cuyos diagramas carga-deformación se distinguen claramente del grupo de resistencia intermedia, en el que se encuentran los tiempos de exposición de 4, 6, 8 y 10 minutos. El diagrama para $t_{\exp }=12$ minutos corresponde al tercer grupo, de resistencia baja, que se separa igualmente del grupo intermedio.

Este fenómeno se observa con mayor detalle en la Figura 8b, la cual presenta los segmentos lineales de los diagramas carga-deformación de los siete grupos. Las magnitudes de estas zonas elásticas se sitúan por debajo de una carga de $4000 \mathrm{~N}$ y corresponden a una deformación menor a $0,005 \mathrm{~m}$. Además, las pendientes $\Delta \mathrm{P} /$ $\Delta y$ se agrupan de manera similar a las tendencias del comportamiento general presentadas en la Figura 8a.

En los resultados anteriores, se observa que la resistencia mecánica de la madera laminada disminuye a medida que la sección transversal disminuye como resultado de la exposición al fuego. Sin embargo, este comportamiento no es proporcional al transcurso del tiempo de exposición. El módulo de elasticidad, empleado aquí como indicador de la resistencia mecánica de la madera, disminuye en intervalos.

La Figura 9 presenta la variación del módulo de elasticidad en función del tiempo de exposición. No obstante que la tendencia general resulta en una correlación lineal con un fuerte coeficiente de determinación, la observación a detalle muestra que el módulo de elasticidad desciende para los tiempos de exposición correspondientes a 0,2 y 4 minutos. Este último tiempo de exposición es el punto de inflexión para una tendencia menos pronunciada en la

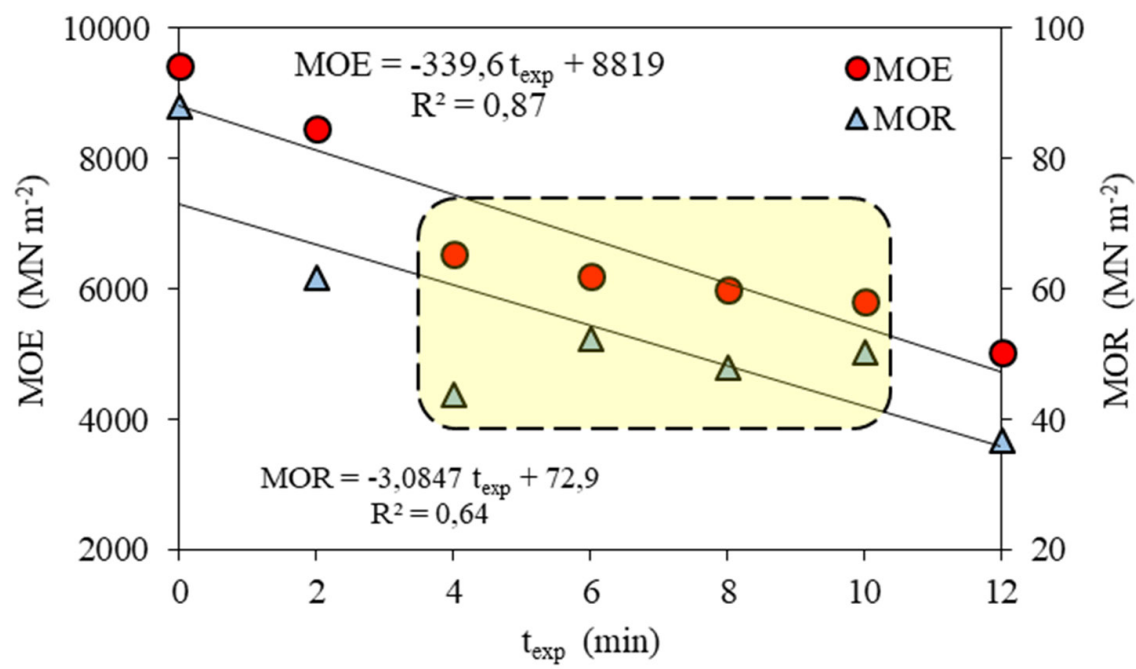

Figura 9. Variación del módulo de elasticidad (MOE) y del módulo de ruptura (MOR) en función del tiempo de exposición $\left(\mathrm{t}_{\exp }\right)$. 


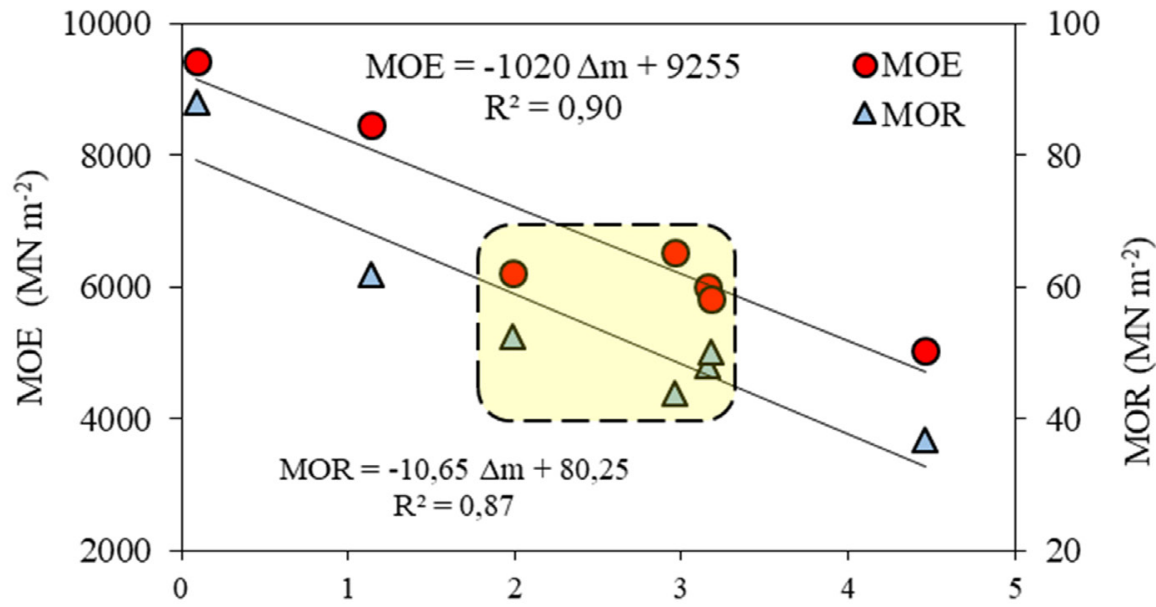

Figura 10. Módulo de elasticidad (MOE) y módulo de ruptura (MOR) en función de la pérdida de masa $(\Delta \mathrm{m})$.

disminución del módulo de elasticidad para los tiempos siguientes de 6,8 y 10 minutos, para finalmente continuar el descenso al minuto 12.

Estos resultados pueden relacionarse con las tendencias de la disminución de las dimensiones de la sección transversal (Figura 6) y con las de la pérdida de masa (Figura 7), ambos parámetros en función del tiempo de exposición. Efectivamente, la Figura 10 presenta la variación del módulo de elasticidad en función de la pérdida de masa. No obstante que la correlación lineal entre estas dos variables presenta un fuerte coeficiente de determinación, la tendencia del módulo de elasticidad a disminuir, en relación con la pérdida de masa de las probetas, se detiene durante el periodo correspondiente a los tiempos de exposición 4 , 6,8 y 10 minutos. Este resultado confirma la uniformidad de los grupos homogéneos para las disminuciones del módulo de elasticidad correspondientes a estos tiempos (Cuadro 2).

\section{Módulo de ruptura}

El módulo de ruptura promedio de las probetas de madera laminada que no fueron expuestas el fuego fue 53,7 \% mayor que el módulo de ruptura de madera sólida de $P$. pseudostrobus $\left(\mathrm{MOR}=57,27 \mathrm{MN} \cdot \mathrm{m}^{-2} ; \rho_{\mathrm{CH}}=540 \mathrm{~kg} \cdot \mathrm{m}^{-3}\right)$ reportada por Sotomayor (2015). El módulo de ruptura de esta investigación es mayor en comparación con los valores declarados por el Laboratorio de Productos Forestales de los Estados Unidos (Forest Products Laboratory 2010) para madera estructural laminada, los cuales van de 28,61 MN.m ${ }^{-2}$ hasta 62,62 MN.m ${ }^{-2}(400$ kg. $\left.\mathrm{m}^{-3}<\rho_{\mathrm{CH}}<600 \mathrm{~kg} \cdot \mathrm{m}^{-3}\right)$.

El módulo de ruptura disminuye a medida que el tiempo de exposición aumenta. De manera análoga al comportamiento del módulo de elasticidad, el del módulo de ruptura se puede asociar a la disminución de la sección transversal de las probetas y a su correspondiente pérdida de masa.

La Figura 9 presenta la variación de módulo de ruptura, en función del tiempo de exposición. La correlación lineal resulta con un coeficiente de determinación moderado. Ahí se señala el intervalo de los valores medios de cinco mediciones en cada muestra del módulo de ruptura, correspondiente a los tiempos de exposición de 4, 6, 8 y 10 minutos. Durante este lapso, después de disminuir comparativamente en los tiempos de exposición 0 y 2 minutos, la disminución se detiene y no decrece necesariamente para los tiempos de exposición 4, 6, 8 y 10 minutos.

En el mismo contexto, el módulo de ruptura correlaciona linealmente con la pérdida de 
Disminución de la resistencia mecánica de madera laminada ocasionada por su exposición al fuego

masa y presenta un fuerte coeficiente de determinación (Figura 10). Desde esta perspectiva, se observa el intervalo de valores de la pérdida de masa, correspondientes a los tiempos de exposición de 4, 6, 8 y 10 minutos. En este intervalo, los valores promedio del módulo de ruptura son similares. A partir del minuto 4, la tendencia de los valores promedio a disminuir, se paran para continuar su descenso en el minuto 12. Este resultado coincide con las observaciones del análisis de grupos homogéneos.

\section{Conclusiones}

La disminución de la masa de madera laminada de $P$. pseudostrobus aumenta en función del tiempo de exposición de la madera al fuego. En cambio, las magnitudes de los módulos de elasticidad y de ruptura disminuyen a medida que transcurre el tiempo de exposición al fuego. Sin embargo, durante el proceso de combustión de la madera, la disminución de su masa, así como de sus módulos de elasticidad y de ruptura, exhibe una pauta entre dos y diez minutos. Los parámetros determinados correlacionan estadísticamente de manera importante con el tiempo de exposición medido. Sin embargo, los resultados son particulares a la madera laminada de P. pseudostrobus y para las condiciones experimentales de esta investigación.

Se fortalece el paradigma vigente en ingeniería de la madera el cual sostiene que después de un periodo de exposición al fuego, la madera crea una capa de madera carbonizada que actúa como protección y aislante, de tal forma que desacelera el proceso de reducción de la sección transversal de una pieza de madera y su pérdida de masa. Consecuentemente, su rigidez mecánica se desacelera durante el proceso de exposición al fuego, en este caso representada por la combinación del momento de inercia de la sección trasversal y sus módulos de elasticidad y de ruptura.

\section{Agradecimientos}

A los alumnos de la Facultad de Ingeniería en Tecnología de la Madera de la Universidad Michoacana de San Nicolás de Hidalgo, Mé- xico, por su participación en la preparación de las probetas. La investigación estuvo patrocinada por la Coordinación Científica de la misma Universidad.

\section{Bibliografía}

Almeida, G; Santos, DVB; Perré, P. 2014. Mild pyrolysis of fast-growing wood species (Caribbean pine and Rose gum): Dimensional changes predicted by the global mass loss. Biomass and Bioenergy 70:407-415.

Bartlett, AI; Hadden, RM; Hidalgo, JP; Santamaria, S; Wiesner, F; Bisby, LA; Deeny, S; Lane, B. 2017. Auto-extinction of engineered timber: Application to compartment fires with exposed timber surfaces. Fire Safety Journal 91:407-413.

Bednarek, Z; Griškevičius, M; Šaučiuvėnas, G. 2009. Tensile, Compressive and Flexural Strength Reduction of Timber in Fire. Statybinès Konstrukcijos ir Technologijos 1(3):148-156.

Brandon, D; Schmid, J; Just, A. 2016. Eurocode 5 design in comparison with fire resistance tests of unprotected timber beams. Conference on performance based design and fire safety design methods SFPE $(11,2016$, Warsaw, Poland). Warsaw, Poland.

Čekovská, H; Gaff, M; Osvaldová, LM; Kačik, F; Kaplan, L; Kubs, J. 2017. Tectona grandis Linn. and its Fire Characteristics Affected by the Thermal Modification of Wood. Bioresources 12(2):2805-2817.

Chan-Hom, T; Yamsaengsung, W; Prapagdee, B; Markpin, T; Sombatsompop, N. 2017. Flame retardancy, antifungal efficacies, and physical-mechanical properties for wood/polymer composites containing zinc borate. Fire and Materials 41(6):675-687.

Cueff, G; Mindeguia, JC; Dréan, V; Breysse, D; Auguin, G. 2018. Experimental and numerical study of the thermomechanical behaviour of wood-based panels exposed to fire. Construction and Building Materials 160:668-678.

Elvira-León, JC; Chimenos, JM; Isábal, C; Monton, J; Formosa, J; Haurie, L. 2016. Epsomite as flame retardant treatment for wood: 
Preliminary study. Construction and Building Materials 126:936-942.

Emberley, R; Do, T; Yim, J; Torero, JL. 2017. Critical heat flux and mass loss rate for extinction of flaming combustion of timber. Fire Safety Journal 91:252-258.

Forest Products Laboratory. 2010. Wood handbook. Wood as an engineering material. General Technical Report FPL-GTR-190. U.S. Department of Agriculture, Forest Service, Forest Products Laboratory. Madison, USA. 508 p.

Friquin, KL. 2011. Material properties and external factors influencing the charring rate of solid wood and glue-laminated timber. Fire and Materials 35(5):303-327.

Gernay, T; Franssen, JM. 2015. A performance indicator for structures under natural fire. Engineering Structures 100:94-103.

Hopkin, DJ; El-Rimawi, J; Silberschmidt, V; Lennon, T. 2011. An effective thermal property framework for softwood in parametric design fires: Comparison of the Eurocode 5 parametric charring approach and advanced calculation models. Construction and Building Materials 25(5):2584-2595.

ISO (International Organization for Standardization). 2014a. Wood. Determination of moisture content for physical and mechanical tests. ISO 13061-1:2014. Geneva, Switzerland. 11 p.

ISO (International Organization for Standardization). 2014b. Wood. Determination of density for physical and mechanical tests. ISO 13061-2:2014. Geneva, Switzerland. 11 p.

Kinjo, H; Horio, T; Hirashima, T; Katakura, Y; Saito, K; Yusa, S. 2016. Deflection behaviour and load bearing capacity of larch glued laminated timber beams exposed to standard fire heating during the cooling phase: Study on fire performance of structural glued laminated timber beams. Part 2. Journal of Structural and Construction Engineering 81(726):1355-1361.

König, J. 2009. The reduced cross-section method for light timber frame construction with solid timber members. Borås, Sweden, SP Technical Research Institute of Sweden. 22 p.
Laguarda Mallo, MF; Espinoza, O. 2015. Awareness, perceptions and willingness to adopt Cross-Laminated Timber by the architecture community in the United States. Journal of Cleaner Production 94:198-210.

Leško, R; Lopušniak, M. 2016. Determination of Fire Resistance of Ceiling Structure Variant Design on the Basis of Timber Using Numerical calculation methods. Applied Mechanics and Materials 820:379-384.

Li, Z; He, M; Lam, F; Li, M; Ma, R; Ma, Z. 2014. Finite element modeling and parametric analysis of timber-steel hybrid structures. The Structural Design of Tall and Special Buildings 23(14):1045-1063.

Lineham, SA; Thomson, D; Bartlett, AI; Bisby, LA; Hadden, RM. 2016. Structural response of fire-exposed cross-laminated timber beams under sustained loads. Fire Safety Journal 85:23-34.

Mačiulaitis, R; Jefimovas, A; Zdanevičius, P. 2012. Research of natural wood combustion and charring processes. Journal of Civil Engineering and Management 18(5):631-641.

Mačiulaitis, R; Praniauskas, V; Yakovlev, G. 2013. Research into the fire properties of wood products most frequently used in construction. Journal of Civil Engineering and Management 19(4):573-582.

Missanjo, E; Matsumura, J. 2016. Wood density and mechanical properties of Pinus kesiya Royle ex Gordon in Malawi. Forests 7(7):135144.

Nadir, Y; Nagarajan, P. 2014. The behavior of horizontally glued laminated beams using rubber Wood. Construction and Building Materials 55:398-405.

Ni, Z; Qiu, P. 2012. Experimental study on fire resistance performance of glulam beams. Applied Mechanics and Materials 193-194:539543.

Östman, B; Brandon, D; Frantzich, H. 2017. Fire safety engineering in timber buildings. Fire Safety Journal 91:11-20. 
Polocoșer, T; Kasal, B; Stöckel, F. 2017. Stateof-the-art: intermediate and high strain rate testing of solid wood. Wood Science and Technology 51(6):1479-1534.

Rocha, MA; Landesmann, A. 2016. Combustion properties of Brazilian natural wood species. Fire and Materials 40(2):219-228.

Sáenz, Reyes, JT; Muñoz Flores, HJ; Rueda Sánchez, A. 2011. Especies Promisorias de Clima Templado para Plantaciones Forestales Comerciales en Michoacán. Uruapan, México, Instituto Nacional de Investigaciones Forestales, Agrícolas y Pecuarias. 213 p.

Schmid, J; Just, A; Klippel, M; Fragiacomo, M. 2015. The Reduced Cross-Section Method for Evaluation of the Fire Resistance of Timber Members: Discussion and Determination of the Zero-Strength Layer. Fire Technology 51(6):1285-1309.

Schmid, J; Klippel, M; Just, A; Frangi, A. 2014. Review and analysis of fire resistance tests of timber members in bending, tension and compression with respect to the Reduced Cross-Section Method. Fire Safety Journal 68:81-99.

Sotomayor Castellanos, JR. 2015. Banco FITECMA de características físico-mecánicas de maderas mexicanas. Morelia, México, Universidad Michoacana de San Nicolás de Hidalgo. $65 \mathrm{p}$.

Sotomayor Castellanos, JR. 2016. Módulo dinámico de la madera de Pinus pseudostrobus. Evaluación por vibraciones transversales. Investigación e Ingeniería de la Madera 12(2):3448.

Sotomayor Castellanos, JR. 2017. Determinación por ondas de esfuerzo del módulo dinámico en madera laminada de Pinus pseudostrobus. Revista Ciencia Nicolaita 72(3):118135.

Sotomayor Castellanos, JR; Carmona Delgado, I; Cervantes Móreles, I; Garduño Suárez, D; Jiménez Guzmán, DZ; Lemus Durán, R; Maldonado Correa, D; Pérez Tello, A; Vaca Hernández, MA; Valdez Velázquez, O. 2015.
Madera laminada de Pinus pseudostrobus. Caracterización dinámica con métodos no destructivos. Investigación e Ingeniería de la Madera 11(3):4-34.

Sotomayor Castellanos, JR; Gallegos León, G. 2018. Reacción al fuego de madera sólida y laminada de Enterolobium cyclocarpum, Tabebuia rosea y Juniperus pyriformis. Estudio comparativo. Investigación e Ingeniería de la Madera 14(1):39-78.

Wiesner, F; Bisby, L. 2018. The structural capacity of laminated timber compression elements in fire: A meta-analysis. Fire Safety Journal. DOI: https://doi.org/10.1016/j.fire$\underline{\text { saf.2018.04.009 }}$

$\mathrm{Xu}, \mathrm{Q}$; Chen, L; Harries, KA; Zhang, F; Liu, Q; Feng, J. 2015. Combustion and charring properties of five common constructional wood species from cone calorimeter tests. Construction and Building Materials 96:416-427.

Yuksel, M; Baysal, E; Toker, H. 2014. Combustion characteristics of oriental beech wood impregnated with commonly used borates. Wood Research 59(1):39-50.

Zhao, Z; Ma, Q; Mu, J; Yi, S; He, Z. 2017. Numerical analysis of Eucalyptus grandis $\times$ E. urophylla heat-treatment: A dynamically detecting method of mass loss during the process. Results in Physics 7: 5-15. 